\title{
Hair hear!
}

At the end of 1996, when only one nuclear gene had been implicated in non-syndromic (NS) deafness ${ }^{1}$, Christine Petit surmised that while progress on the genetic dissection of deafness had been slow for a variety of reasons, persistent efforts would soon yield fruit ${ }^{2}$. This has turned out to be an accurate prediction; no fewer than six 'NS' genes have been identified in the meantime $e^{3-8}$. Three of these are implicated in maintaining cytoskeletal integrity and have provided grist for the mill of conjecture regarding their specific roles in the inner ear.

Myo7a (encoding myosin VIIa) has a head start on the other 'NS' genes, as it was also implicated in syndromic deafness (both in mouse and human) in 1995 (refs 9,10). A recent analysis of mutant mice that carry three 'non-syndromic' versions of mutated Myo7a has demonstrated that its gene product is essential for the maintenance and organization of the stereocilia that project from the sensoryhair cells in the organ of Corti (see figure; ref. 11). Furthermore, the 'degree' of mutation affects the phenotype accordingly, with the most 'severe' mutation effecting the deafest mouse. But what does myosin VIIa actually do in the sensory-hair cells? Electron microscopy of inner hair cells from wild-type and mutant mice suggests that while the stereocilia, which are essentially specialized villi, develop normally in the mutants, something happens during their development that renders them unable to maintain their orientation, both with respect to one another and with respect to the cuticular plate (a submembranous structure composed of a dense network of actin fibers, and in which wild-type stereocilia anchor via 'rootlets'). In fact, the cuticular plate itself is disorganized in mutants, and rather than inserting into it, the stereocilia sometimes insert into vesicledense regions between the cuticular and surface membranes, or between 'islands' of cuticular plate. Furthermore, the kinocilium, a long cilium that normally appears at the apex of the V-shaped stereocilia formation (see figure), appears to have no spatial relationship with its disorganized brethren in the two more severe mutants, leading the investigators to conclude that either the kinocilium requires myosin VIIa to organize them, or the kinocilium does not normally play a role in their organization. Another means by which perturbation of myosin function may affect hearing is via the 'tip links' - extracellular bridges that link the tip of a smaller stereocilium with the apical region of its taller neighbour. When these links are stressed, as would be the case when a sound-generated vibration travels through the fluid of the inner ear, altering the physical position of the basilar membrane (to which the sensory hair cells are indirectly anchored) relative to that of the tectorial membrane (against which the ends of the sterocilia rest), transduction channels in the stereocilium open, leading to membrane depolarization. It is thought that a molecular motor, perhaps composed of myosin and probably located at the end of the tip link that is embedded in the taller stereocilium, modulates tip-link tension (which, in turn, affects transduction channels), by slipping down or ratcheting up the actin cytoskeleton. There is a wealth of hypotheses for myosin VIIa action in the inner ear, which will not be honed down without functional studies and further characterization of protein expression.

Mutations in DFNA1 (it is a homologue of Drosophila diaphanous and has yet to be designated an approved symbol), reported by Eric Lynch and colleagues last November ${ }^{6}$, are also

Hair and now? Stereocilia normally project in a three-tiered, V-shapped pattern $(\mathbf{a}, \mathbf{c})$ from the apical surfaces of sensory hair cells in the inner ear (see ref. 1). In contrast, mutant mice carrying mutant myosin Vlla have disorganized sterocilia bundles $(\boldsymbol{b}, \boldsymbol{d})$. Image kindly provided by Tim Self and Karen Steel ${ }^{11}$, and reproduced with permission from The Company of Biologists.

likely to affect cytoskeletal integrity. The products of its yeast and Drosophila homologues interact with proteins known to polymerize actin in such a way as to directly implicate it in this process. While the characterization of mutant phenotypes has yet to be reported, it seems likely that the integrity of the cuticular membrane or the stereocilia, which also contain crosslinked actin filaments, will be affected.

As reported by Kristien Verhoeven on page 60 of this issue, TECTA (encoding $\alpha$-tectorin,) now joins the fray ${ }^{12}$. As its name suggests, $\alpha$-tectorin is a component of the tectorial membrane, an acellular, comparatively featureless membrane that has received much less attention than the organ of Corti, with its aesthetic and intricate components. TECTA is also the first 'NS' gene whose expression is believed to be restricted to the organ affected; the other 'NS' genes are widely expressed. Its product is processed into three cross-linked polypeptides that contribute to the non-collagenous matrix of the tectorial membrane - it is not hard to envisage how alterations in membrane consistency would affect sensory signal transmitted by the abutting stereocilia.

There are many experimental miles to go before the workings of the inner ear - in sickness and in health - are understood, but it is clear that the discovery of 'deafness' mutations will continue to deliver mechanistic insight, if not accurate aural sensitivity. The accrual of these mutations is literally hairing ahead.

\section{- Bette Phimister}
1. de Kok, Y.J.M. et al. Science 278, 685-688 (1995).
2. Petit, C. Nature Genet. 14, 385-391 (1996).
3. Kelsell, D.P. et al. Nature 387, 80-83 (1997).
4. Liu, X.-Z. et al. Nature Genet. 16, 188-190 (1997).
5. Weil, D. et al. Nature Genet. 16, 191-193 (1997)
6. Lynch, E.D. et al. Science 278, 1315-1318 (1997).
7. Avraham, K.B. et al. Science 279, 1950-1954 (1998)
8. Li, X. et al. Nature Genet. 18, 215-218 (1998).
9. Gibson, F. et al. Nature 374, 62-64 (1995).
10. Weil, D. et al. Nature 374, 60-61 (1995).
11. Self, T. et al. Development 125, 557-566 (1998).
12. Verhoeven, K. et al. Nature Genet. 19, 60-63 (1998). 